\title{
Subject Index Vol. 31, 1994
}

\section{A23187 92}

Acetylcholine 18,92, 187 Adrenergic nerve function 25 ß-Adrenoceptors 18 Alcianblue 2

Allylamine 33 Angiogenesis 178,195,205 Angioplasty 153 Angiotensin 338

receptors 338 Angiotensin-II 144, 163

receptor 10 Angiotensin-converting enzyme

52,265 Antisense oligonucleotide 322

$$
\text { - } \quad \text { RNA } 178
$$

Aorta 33,256

-, rat 52

Arginine vasopressin 144 Arterial injury 247

- wall 322

Arteries, human 280

Arteriolar diameter 287

Atherosclerosis 61, 173, 187,

280, 347 ATP sensitivity 256 ATP-sensitive K+ channels 82 Autonomic neuropathy 25

$\mathrm{Ba} 2+$ currents 256

Basement membrane synthesis

195 Basilar artery 106 Blood flow 187

- $\quad$ pressure 347

Bromodeoxyuridine 247

$\mathrm{Ca} 2+$ agonist 256

antagonist 256

channels 359

-, currents 271

influx 256

Calcium 82,265,314,330

channels 240,314 cAMP 195

analogs 121 cAMP-dependent protein

kinase 121,271 Capillary 205 Captopril 52

Cardiovascular system 131 Caudal artery 25 Cell culture 330

cycle kinetics 247

- time 230

density 330

growth 330

proliferation 230, 280 Cerebral arteries 221

blood flow 221 cGMP 330 cGMP-dependent protein

kinase 271 Collagen, type I 280 Colloidal gold 10 Contraction 92 Coronary artery 338 Cyanide 82 Cyclic AMP 42 Cyclopiazonic acid 18 
Deoxycorticosterone 347 Desmopressin 216 Diabetes mellitus 25

Echo-tracking system 114 Electron microscopy 10 Electrophysiology 82 Endothelial cells

131,178,230 Endothelin 1144 Endothelium 2,18,92,131,

173,205,221 Endothelium-derived relaxing

factor 18, 131 Expression 330 Extracellular matrix 178

Female 106 Fluorescent lectins 2 Forskolin 18 Free radicals 33 Fura-2 265

Gap junctions 307 Glibenclamide 82 Glyceryl trinitrate 187 Glycocalyx 2

Hamster cheek pouch 287

Heart 33

Heptanol 307

Human umbilical artery 92 ,

240 Hybridisation in situ 322 Hydroxyl radical generation 33 Hypertension 52,61,359

Hypertonicity 240 Hypotonicity 240

Inactivation recovery 359 Intercellular communication 307 Intima-media thickness 114 Intimal

hyperplasia 153 Intracellular calcium 144

- $\quad$ signalling 195

Isoforms, myosin 61

Isoproterenol 18

$\mathrm{K}+$ channels 256

L. and T-type channels 359 Lr Arginine 131 Laminin isoforms 163 Lipid peroxidation 33

Macrovascular endothelial cells

230 Male 106 Membrane(s) 359

potential 314

N-Methyl-Z > -aspartate recep

tors 221

Methylene blue 18 Microcirculation 287 Microvascular endothelial cells

230 Microvessels 338 Mitochondria 33 MK-801 221 Myosin 61

Nasal vasculature 216 Neo-intima 153, 187 Neovascularization 195 New Zealand White 106

Nitric oxide 42,52,131,187, 221

- $\quad$ - synthase 131

NG-Nitro-L-arginine methyl-

ester 18

$\mathrm{N} \omega$-Nitro-L-arginine 52 Nitroprusside 153 Noradrenaline 25 -, rat 314

Oxidative stress 33 Oxygen tension 92

Phenotype 330 Phenylephrine 153 Phosphorylation 330 Platelet-activating factor 144 Portal vein smooth muscle 271 Procollagen 280

Proliferating cell nuclear

antigen 280 Protein kinase 330

- $\quad$ - C 195

Proteoglycan inhibition 322

Quantitative fluorescence microscopy 2

Radial artery 114 Receptor-mediated endocytosis

10 Regulation 131 Relaxation 92, 121 Resistance, nasal 216

- $\quad$ vessels 187

Restenosis 153

Secretion, nasal 216 
Smooth muscle cell(s) 61, 153,

330 Sodium nitroprusside 18 Spontaneously hypertensive rat

52 Stathmokinetic index 230

Tetraethylammonium 307 Thiol content 33 Thoracic aorta 18 Thrombin 42, 173

Thrombospondin 178 Thromboxane 52 Time-lapse microcinemato-

graphy 230 Transendothelial electrical

resistance 42 Transforming growth factor- $\beta \mathbf{i}$

322

Vascular diseases 61

- $\quad$ reactivity 347

smooth muscle $18,82,121,247,265,307,314$

- - cells 144,163

- - tissue 61

- $\quad$ tone 82

Vasculogenesis 205

Vasodilation 256

Vasomotion, arteriolar 287

Vasopressin 216

Watanabe heritable hyperlipid-

(a)emic rabbit(s) 106, 347 Whole-cell voltage clamp 271 Wistar Kyoto rat 52 370 